\title{
Art ou ethnologie? Questions de présentation dans les Museen für Völkerkunde en Allemagne après 1900
}

Anne-Solène Rolland

\section{OpenEdition}

\section{Journals}

Édition électronique

URL : http://journals.openedition.org/actesbranly/155

DOI : 10.4000/actesbranly. 155

ISSN : 2105-2735

Éditeur

Musée du quai Branly Jacques Chirac

Référence électronique

Anne-Solène Rolland, « Art ou ethnologie? Questions de présentation dans les Museen für Völkerkunde en Allemagne après 1900 », Les actes de colloques du musée du quai Branly Jacques Chirac [En ligne], 1 | 2009, mis en ligne le 28 juillet 2009, consulté le 08 septembre 2020. URL : http://

journals.openedition.org/actesbranly/155; DOI : https://doi.org/10.4000/actesbranly.155

Ce document a été généré automatiquement le 8 septembre 2020

(C) Tous droits réservés 


\title{
Art ou ethnologie? Questions de présentation dans les Museen für Völkerkunde en Allemagne après 1900
}

\author{
Anne-Solène Rolland
}

1 L'Allemagne est sans doute le pays d'Europe où les collections ethnologiques, encore aujourd'hui, sont les plus nombreuses. Héritiers de cabinets de curiosité des souverains locaux ou de particuliers, des musées d'ethnologie sont créés dans toutes les grandes villes d'Allemagne à partir de 1860 sous l'impulsion de scientifiques passionnés, venus de la médecine (comme Ad Bastian à Berlin ou Adolf Bernhard Meyer à Dresde) ou des sciences naturelles (Moritz Wagner, géologue, à Munich). Un Museum für Völkerkunde est créé en 1868 à Munich, en 1869 à Leipzig, en 1873 à Berlin, en 1875 à Dresde, en 1879 à Hambourg, pour ne citer que les plus importants. Le musée de Berlin est le premier conçu comme musée d'Ethnologie indépendant. L'idée maitresse de son fondateur, Adolf Bastian (1826-1905), est de collecter les derniers témoignages matériels de civilisations naturelles menacées par l'avancée de la culture européenne: «ce qui disparaît maintenant est perdu à jamais pour nos successeurs ", écrit Bastian en $1876^{1}$. Le musée doit donc recueillir et étudier ces témoignages afin d'améliorer la connaissance générale de l'homme. Bastian, comme beaucoup après lui, est en effet persuadé de l'existence d'une "unité psychique de l'homme": la comparaison des objets de tous les peuples doit amener les scientifiques à la découverte de ces éléments communs à toute l'humanité. Les musées ethnologiques, à Berlin comme ailleurs, sont donc avant tout des instruments au service de la science et de la connaissance de l'homme. Au tournant du siècle, ces musées de science, bien implantés, sont les fers de lance d'une ethnologie qui s'est peu à peu imposée comme science.

2 Les premières années du xxe siècle marquent une rupture dans cette conception scientifique des musées. Beaucoup d'entre eux, à l'étroit dans leurs bâtiments d'origine, sont réaménagés. À cette occasion, de nouvelles conceptions muséographiques 
apparaissent, qui incluent une réflexion sur la valeur esthétique et artistique des collections. Cette réflexion s'inscrit dans un mouvement plus général, particulièrement fort en Allemagne, qui reconsidère la nature des collections extra-européennes. À l'exemple d'August Macke et Vassily Kandinsky du Blaue Reiter munichois, les artistes des avant-gardes allemandes s'intéressent aux objets exotiques; les historiens d'art Alois Riegl et Ernst Grosse les intègrent à leurs théories de l'histoire de l'art, tandis que des ethnologues comme Karl von den Steinen s'intéressent à la question de l'ornementation. De nouveaux points de vue apparaissent et les regards sur les objets se démultiplient. L'art, finalement, apparaît dans les musées d'ethnologie².

3 Au début du xxe siècle, la séparation des collections en Schausammlungen, collections présentées au public et Studiensammlungen, collections d'études, oblige les ethnologues à réfléchir à la dimension esthétique de leurs objets. Les rénovations architecturales permettent aussi de repenser la mise en scène des objets, qui devient plus esthétisante. Là où certains, comme Adolf Bastian, sont formellement opposés à ces nouvelles orientations, d'autres veulent aller plus loin encore, et prônent la création de musées à part pour les cultures dites artistiques : en Allemagne comme ailleurs, le débat sur la place de l'art dans les cultures non-européennes se focalise sur les collections asiatiques. Lucian Scherman, directeur du musée de Munich à partir de 1907, incarne une forme de compromis entre les positions des ethnologues et celles des défenseurs de l'art, faisant de la rénovation du musée de Munich un exemple inédit et très remarqué.

4 Schausammlungen et Studiensammlungen dans les Museen für Völkerkunde : de la science à l'esthétique - les principaux facteurs de la séparation des collections

5 Partout, à l'orée du xxe siècle, les musées d'ethnologie sont dépassés par leur succès. Ils ont considérablement enrichi leurs collections, si bien que les bâtiments ne sont plus assez grands. Le musée de Berlin, « où l'on se sent perdu, parce que dans les couloirs sans murs s'entassent vitrines et placards [...], [est] devenu un chaos incroyable $»^{3}$.

6 En 1907, le directeur général des musées de Berlin, Wilhelm Bode, décide la construction d'un nouvel édifice, trente ans seulement après l'inauguration du bâtiment mis en cause. Comme à Berlin, nombreux sont les musées dont les collections sont réorganisées ou qui déménagent ${ }^{4}$. La Première Guerre mondiale marque l'arrêt de tous ces projets. Mais dès cette époque, matériellement, les principes scientifiques des musées d'ethnologie sont mis à mal.

7 La question du public des musées s'ajoute à ces problèmes matériels. Comme l'a bien analysé H. Glenn Penny ${ }^{5}$, elle est devenue prégnante au début du xxe siècle. Le rôle des musées en général est à cette époque envisagé différemment : propriétaires et mécènes souhaitent en faire des institutions visibles, destinées à tous et qui bénéficient/ contribuent à leur image. Les musées de tout genre, dont la mission principale était la collecte exhaustive et l'étude des collections, sont invités à prendre une place plus importante dans la vie sociale de leurs villes. Ils doivent désormais s'adresser aux classes moyennes qui émergent de la révolution industrielle allemande et souhaitent avoir accès à la culture.

8 La question que se posent alors les ethnologues est de savoir comment concilier une science ethnologique en plein essor, construite autour des collections muséales, et une présentation qui convienne au plus grand nombre. Presque partout, ils commencent par séparer les collections: d'un côté les Schausammlungen, collections présentées au 
public, de l'autre les Studiensammlungen, collections d'études, accessibles aux seuls chercheurs.

\section{La création de Schausammlungen : la défaite de la science}

9 Les musées d'ethnologie reprennent cette idée des musées d'histoire naturelle, en particulier du musée d'histoire naturelle de Londres. Il s'agit d'une rupture conceptuelle considérable. En effet, les ethnologues, pour aménager les nouvelles galeries, doivent sélectionner les objets qu'ils souhaitent présenter au public, ce qui est à l'opposé de l'ethnologie de la totalité de Bastian. Pour la première fois, les ethnologues prennent en compte des objets individualisés et non plus des ensembles: par le choix de l'objet à exposer, c'est aussi le regard de l'ethnologue sur l'objet qui change. Oswald Richter, conservateur à Dresde, justifie ainsi la séparation par le plaisir du visiteur. Selon lui, « l'intérêt croissant du public pour l'art [obligera les musées] à l'avenir à prendre plus en compte la valeur esthétique de l'objet ethnographique, c'està-dire sa qualité esthétique, sa valeur en tant qu'œuvre d'art $»^{6}$.

brèche est ouverte dans l'approche purement scientifique. Comme le souligne Andrew Zimmerman, « l'opposition binaire art primitif/objet de science était l'une des oppositions qui était à la base même de la possibilité d'une anthropologie comme science naturelle de l'humanité $»^{7}$ : par la conception de Schausammlungen et l'introduction de l'idée d'esthétique dans les musées d'ethnologie, les fondements de l'ethnologie sont ébranlés. Les résistances sont nombreuses, notamment au musée de Berlin où une polémique fait rage dès 1900 . En son sein même, n'ignorant pas les discussions de leurs collègues des autres musées, certains ethnologues souhaitent réorganiser les collections. Felix von Luschan, responsable des collections africaines, prône très tôt l'aménagement de Schausammlungen :

« Pour moi, il serait tout à fait nécessaire [...] de distinguer une collection présentée au grand public ", écrit-il dès 1900. Puis, en 1903: "La séparation d'une Schausammlung peut bien plutôt avoir pour objectif de faciliter la compréhension des collections pour le public inéduqué8." "

11 Mais sa position est minoritaire. Les musées d'ethnologie, expliquent ses collègues, n'ont pas pour vocation « la satisfaction du voyeurisme du public »'. La mort de Bastian en 1905 affaiblit leur position. En 1910, Bode commande de nouveaux plans pour le musée d'ethnologie, qui entérinent la séparation des collections en Schausammlungen et Studiensamlungen. Le nouveau musée, dont la construction est retardée par la Grande Guerre, est inauguré en 1926. Ce commentaire élogieux de l'ethnologue Fritz Krause en 1928 confirme la défaite posthume de Bastian :

« À Berlin et Munich, cet aspect esthétique est mis au premier plan [...]. ÀBerlin, la valeur esthétique de l'objet est même le critère décisif du choix ${ }^{10}$.»

\section{La mise en scène des collections dans des musées rénovés : vers des musées d'art}

12 En pratique, les ethnologues doivent repenser la muséographie pour aménager leurs Schausammlungen. Les musées d'ethnologie privilégiaient l'accumulation. De grandes vitrines permettaient d'exposer un maximum d'objets ${ }^{11}$. Alignées en rangées parallèles 
serrées, elles emplissaient les salles des musées et permettaient au regard d'embrasser un maximum de pièces, obligeant au regard comparatif de l'ethnologie et interdisant le regard individualisant de l'art. Les Schausammlungen et le plaisir du visiteur appellent précisément le contraire : le style adopté est celui de l'isolation des objets dans de plus petites vitrines, afin que « chaque pièce individuellement puisse exprimer entièrement et seulement sa puissance esthétique $»^{12}$.

C'est à Munich que les changements muséographiques sont les plus importants. Sous l'impulsion de Scherman, qui prend la tête du musée en 1907, les salles se transforment radicalement ${ }^{13}$. Il intervient sur le mobilier des salles. Il remplace les tables sur lesquelles étaient accumulés les objets par des armoires à étagères: la verticalité permet une meilleure visibilité. Il présente certains d'entre eux sans vitrine afin de les mettre mieux en valeur. Il s'attache aussi à l'aspect d'ensemble des salles : des miroirs permettent de multiplier les points de vue sur les objets et de donner plus de profondeur aux salles. Surtout, il introduit l'usage des couleurs dans la décoration des salles. Il propose donc une vision muséographique globale, qui prend en compte la beauté des objets et le plaisir du visiteur.

Mais Scherman va plus loin. Il associe des artistes au réaménagement, puis au déménagement, en 1923, du musée: le peintre d'histoire suisse Hans Eduard von Berlepsch-Valendas (1849-1921), assisté par le graveur Oscar Graf (1870-1957) et le peintre Martin Kurreck (1886-1957), sont chargés de la décoration intérieure des bâtiments. Le geste de Scherman est inattendu et tout à fait contraire aux principes de l'ethnologie. Pour la première fois, un musée d'ethnologie est aménagé comme un musée d'art, dans un but avoué d'agrément autant que d'éducation. C'est un changement très net et qui, précisément, est le fait d'un des premiers directeurs de musée d'ethnologie non ethnologue- Scherman était linguiste -, dans une ville très attachée à l'art et à la culture. Les éloges sont nombreux. L'égyptologue Von Bissing écrit ainsi en 1908 :

«Les objets asiatiques, devant des murs aux beaux effets de couleurs, captent l'œil du visiteur dès son entrée, et attirent son attention sur un point fort du musée, l'art de la Chine et du Japon : leur présentation artistique fut de tous côtés applaudie ${ }^{14}$ ».

Surtout, le sens artistique dont fait preuve Scherman dès son arrivée renouvelle l'intérêt des Munichois pour le musée : il trouve par exemple dans le critique d'art Wilhelm Hausenstein, ami de Paul Klee et défenseur du Blaue Reiter, l'un des plus fervents avocats du musée ${ }^{15}$.

\section{Sortir l'art des musées d'ethnologie ? Naturvölker contre Kulturvölker : pour des musées d'art asiatique}

16 Les aménagements de Scherman marquent déjà une rupture importante avec les musées d'ethnologie traditionnels. Certains veulent aller plus loin et séparer objets d'art et objets ethnologiques. Des musées d'art extra-européen existaient, de fait, depuis la fin du xixe siècle : les musées d'art asiatique. Les objets asiatiques ont très tôt bénéficié d'un traitement différent de celui réservé aux autres continents. Il n'est d'ailleurs pas anodin que Scherman ait été lui-même un spécialiste de l'Inde, ni que le point fort du musée de Munich, grâce notamment à une importante donation de 
Philip Franz von Siebold ${ }^{16}$, ait été l'art d'Extrême-Orient. Les arts d'Asie cristallisent ainsi la question de la place de l'art dans les musées d'ethnologie.

À Paris, deux musées d'art asiatique existaient depuis la fin du xixe siècle : le musée Guimet, ouvert en 1889, et le musée Cernuschi, ouvert en 1898. À Vienne, un musée oriental avait vu le jour à la suite de l'Exposition internationale de 1873. Au tournant du siècle, de nombreuses voix s'élèvent en Allemagne en faveur de tels musées. Deux textes parus dans le premier numéro de la revue Museumskunde en 1905 font date. Ernst Grosse ${ }^{17}$, sinologue, directeur des collections de Fribourg, et Woldemar von Seidlitz, directeur des collections royales de Dresde ${ }^{18}$, se plaignent de la mauvaise présentation des collections asiatiques en Allemagne. Von Seidlitz insiste très fortement sur l'«égale valeur des visées artistiques et scientifiques» dans la présentation des collections. Il en appelle donc à la séparation des collections asiatiques d'une part, des collections des peuples naturels d'autre part, et à la création d'un musée asiatique à Berlin.

C'est encore une fois à Berlin que les débats sont les plus houleux. Les défenseurs d'un musée asiatique invoquent l'opposition entre Kultur et Natur: de manière générale, Chine, Japon, Asie du Sud-Est et monde islamisé sont admis du côté des peuples de culture, les autres appartenant encore au monde de la nature ${ }^{19}$. C'est pourquoi Augustin Krämer ${ }^{20}$ propose de créer à Berlin deux musées différents, l'un de Kulturvölkerkunde, présentant les cultures bouddhiques et islamiques, l'autre de Naturvölkerkunde, comprenant tous les autres continents et les peuples naturels d'Asie. Pour Fritz Gräbner, ethnologue de Cologne, cet argument plaide justement contre la séparation. Perdre les collections du continent asiatique, source des plus importantes influences culturelles, reviendrait à un anéantissement de l'ethnologie comparative ${ }^{21}$. La création d'un musée asiatique est finalement entérinée dans les plans de déménagement du musée dès 1912. Le musée d'art asiatique est ouvert sous le nom d'Ostasiatische Kunstabteilungle 26 juin 1926 dans le centre de Berlin, au cœur du bâtiment mitoyen du musée d'ethnologie. Les collections asiatiques sont réparties entre les deux musées, selon leur "valeur » : les pièces considérées comme artistiques sont attribuées au musée d'art, les pièces considérées comme intéressantes du point de vue de l'histoire culturelle vont à la section asiatique du musée d'ethnologie. En 1926, le musée exhaustif et comparatif de Bastian, transformé par une double séparation de ses collections, a définitivement vécu.

\section{La question des « arts décoratifs »}

19 Sans aller aussi loin que ceux que Scherman appelle les "fanatiques de l'art $»^{22}$, d'autres rapprochent les collections asiatiques des collections d'arts décoratifs. Ce troisième point de vue vient nourrir la réflexion sur la distinction entre art et ethnologie.

De grands musées d'arts décoratifs, alors appelés d'art et d'industrie (en Allemagne, Kunstgewerbemuseen ou Museen für Kunst und Industrie), ont été fondés en Europe dans les mêmes années que les musées d'ethnologie. Sur le modèle de celui de Londres, aujourd'hui Victoria and Albert Museum, daté de 1852, furent créés le musée de Vienne (1863) puis ceux de Berlin (1867) et de Hambourg (1874). De manière générale, les artistes et les historiens d'art de l'ère industrielle commencent à s'intéresser à des formes d'art nouvelles, considérées comme mineures, liées à la vie quotidienne et où 
l'aspect décoratif prime. Dans cette optique, certains objets exotiques particulièrement décoratifs suscitent un intérêt nouveau : laques et céramiques d'Extrême-Orient, voire objets maoris, dont les circonvolutions attirent l'œil, sont présentés dans ces musées de l'ornement. Le musée de Hambourg, sous l'impulsion de son fondateur Justus Brinckmann, se spécialise ainsi dans les arts décoratifs japonais et présente l'une des plus importantes collections d'Allemagne ${ }^{23}$. Völkerkunde, Kunstgewerbe et Asiatische Kunst constituent alors trois angles d'approche des mêmes objets.

\section{À Munich, tous les aspects de toutes les cultures}

21 Après la Première Guerre mondiale, trois musées présentent des collections extraeuropéennes à Berlin : le musée d'ethnologie, le musée d'art asiatique et le musée des arts décoratifs. À l'opposé de cette dispersion des collections, à Munich, Scherman propose de réunir toutes ces approches dans le nouveau musée d'ethnologie, à partir de 1923.

22 Scherman n'est pas indifférent à l'approche décorative des collections: il veut «maintenir la tradition artistique munichoise en liant étroitement l'ethnologie et l'art et en prenant bien en compte la création artisanale des peuples étrangers ${ }^{24}$. Munich n'a pas de musée des arts décoratifs. Pour Scherman, le musée d'ethnologie doit donc être un lieu d'inspiration pour les artistes et les artisans munichois. Une école d'arts décoratifs est d'ailleurs ouverte, adjointe au musée. Attirer l'attention sur l'aspect ornemental et décoratif lui semble aussi une façon d'intéresser le public à ces objets lointains, en lui permettant des comparaisons avec ce qu'il connaît et en lui rappelant la présentation des musées d'art dont il est plus familier.

Cependant, Scherman adopte sur l'art des positions très différentes de celles de ses confrères berlinois. S'il accepte de présenter les œuvres comme des objets ou des œuvres d'art, ce n'est qu'au sein du musée d'ethnologie. "L'extraction des objets d'art de leur contexte ethnographique [...] ne peut être que nocive ${ }^{25} »:$ il faut donner leur juste place aux "expressions artistiques, en tant que l'un des chapitres les plus importants, peut-être le couronnement, de l'ethnologie $»^{26}$. Scherman, sans être luimême ethnologue, est influencé par les thèses de l'école historico-culturelle, théorisées notamment par Fritz Gräbner et Bernhard Ankermann ${ }^{27}$ : en présentant tous les continents ensemble, il souhaite rendre compte du développement des cultures dans le temps et l'espace, et des relations entre les aires culturelles. Le contenu scientifique n'est donc pas sacrifié à l'esthétique.

Le déménagement du musée lui donne l'occasion de mettre en pratique cette vision globale des collections. Chaque section du nouveau musée est organisée selon une gradation qui va des objets du quotidien aux œuvres d'art exceptionnelles. Ainsi, la section japonaise ${ }^{28}$, l'un des fleurons de la collection munichoise, s'ouvre sur les ustensiles du quotidien; viennent ensuite les salles d'arts décoratifs, qui présentent laques et céramiques, puis objets des cultes quotidiens, peintures et sculptures; enfin, le parcours se termine sur les grandes sculptures bouddhiques, unanimement considérées comme des œuvres d'art. Tout au long de ce parcours, la muséographie s'adapte au contenu : l'ethnographie du début est présentée dans des vitrines denses, les œuvres d'art sont isolées. La réception est excellente : Martin Heydrich décrit le nouveau musée comme une des plus belles expositions d'art et de civilisations « exotiques " ${ }^{29}$. L'ethnologue Fritz Krause écrit: "À Munich, le mélange entre 
l'esthétique et l'histoire des cultures est plus satisfaisant pour l'ethnologue [qu'à Berlin]..$^{30}$ »

C'est ainsi une troisième voie que propose Scherman à Munich : ni science, ni art, mais les deux, pour satisfaire tout le public. N'appartenant ni au camp des ethnologues, ni au camp des historiens de l'art asiatique, il put trouver un compromis.

Les liens entre la science ethnologique et l'approche artistique ont suscité de nombreux débats dans les musées d'ethnologie allemands au début du xxesiècle. Contraints de réaménager leurs collections, les ethnologues réfléchissent à ce qu'ils veulent faire de leurs institutions : c'est ainsi qu'ils se posent, pour la première fois, la question du statut des objets ethnologiques et de leur caractère artistique. Ces réflexions aboutissent à de nouvelles présentations des collections qui introduisent, de manière plus ou moins sensible, la dimension artistique des objets dans les musées ethnologiques. Les exemples des musées de Berlin et Munich, qui sont encore aujourd'hui les deux plus grands musées d'ethnologie allemands, sont particulièrement significatifs de la complexité de ces débats et de la variété des solutions proposées. Là où les musées berlinois développent une opposition définitive des approches scientifiques et artistiques, le musée rénové de Scherman à Munich propose une tentative de réconciliation inédite. Il a marqué une véritable rupture dans l'histoire des musées d'ethnologie, non seulement en Allemagne mais dans toute l'Europe. Herman Frese a souligné l'importance que les prises de position de Scherman à Munich ont eue pour l'apparition des notions d'art primitif et la création de musées d'arts primitifs distincts des musées d'ethnologie ${ }^{31}$.

D'autres musées, comme ceux de Dresde ou Cologne, connurent des évolutions similaires à celles du musée de Munich. Ces évolutions des musées d'ethnologie sont également les signes d'un changement, au début du xxe siècle, dans la réflexion sur le rôle des musées. Le musée exhaustif et scientifique du xixe siècle doit devenir une institution inscrite dans la cité, à vocation sociale et politique. Un musée d'ethnologie qui voulait survivre devait changer et trouver un compromis entre deux positions contradictoires, la rigueur scientifique et l'ouverture au grand public. Les rencontres entre art et ethnologie dans les musées furent ainsi, parfois, très contingentes. Mais il est tout à l'honneur de directeurs comme Scherman d'avoir su, au bon moment, prendre les bonnes mesures pour attirer l'attention des personnes importantes sur les musées d'ethnologie: c'est aussi en cela que l'expérience munichoise, qui mêla ethnologie, art, culture et politique, est significative et moderne.

\section{BIBLIOGRAPHIE}

Ankermann 1905 : Bernhard Ankermann, « Kulturkreise und Kulturschichten in Afrika », Zeitschrift für Ethnologie, 37, 1905, pp 54-84.

Frese 1960 : Hermann Heinrich Frese, Anthropology and the Public : the Role of Museums, Leide, E. J. Brill, 1960. 
Gareis 1990 : Sigrid Gareis, Exotik in München : Museumsethnologische Konzeptionen im historischen Wandel, Munich, Anacon, 1990.

Glenn Penny 2002 : Glenn H. Penny, Objects of Culture, Ethnology and Ethnographic Museums in Imperial Germany, Chapel Hill, The University of North Carolina Press, 2002.

Goldwater 1967 : Robert Goldwater, Primitivism in Modern Art, New York, Random House, 1967. Gräbner 1908 : Fritz Gräbner, « Der Neubau des Berliner Museums für Völkerkunde und andere praktische Zeitfragen der Ethnologie », Globus 94, 1908, p. 213-216

Gräbner 1905 : Gräbner Fritz, « Kulturkreise und Kulturschichten in Ozeanien », Zeitschrift für Ethnologie, 37, 1905, p. 28-53.

Grosse 1905 : Ernst Grosse, « Über den Ausbau und die Aufstellung öffentlicher Samlungen von asiatischen Kunstwerken », Museumskunde, nº, 1905, p. 123-139.

Heydrich 1930 : Martin Heydrich, « La Réorganisation du musée ethnographique de Munich », Mouseion 11, 2, 1930, p. 128-137.

Krämer 1904 : Augustin Krämer, « Der Neubau des Berliner Museum für Völkerkunde im Lichte der ethnographischen Forschung », Globus 86, 1904, p. 21-24

Krause 1920 : Fritz Krause, « Die Wiedereröffnung des Berliner und des Münchener Museums für Völkerkunde », Ethnologischer Anzeiger, nº1, 1928, p. 66-68.

Migge 1967 : Walther Migge, Wilhelm Hausenstein, Wege eines Europäers, Munich, Kösel in Kommission, 1967.

Richter 1906 : Oswald Richter, «Über die idealen und praktischen Aufgaben der ethnologischen Museen », Museumkunde, n'II, 1906, p. 189-218.

Rolland 2008 : Anne-Solène Rolland, « Techniques ou cultures. Histoires croisées des musées d'ethnologie et d'arts décoratifs de Vienne et Hambourg, 1860-1900 ", Histoire de l'art, n 62, avril 2008.

Scheffler 1921 : Karl Scheffler, Der Berliner Museumskrieg, Berlin, Museum War, 1921.

Schmalenbach 1961 : Werner Schmalenbach Die Kunst der Primitiven als Anregungsquelle für die europäische Kunst bis 1900, Cologne, Dumont-Schauberg, 1961.

Scherman 1922 : Lucian Scherman, Kunst im Handwerk abseits der Hochkultur, Neue süddeutsche Illustrierte, 4, Nr 13-15, Munich, 16.8.1922

Smolka 1994 : Wolfgang Smolka, Völkerkunde in München. Voraussetzungen, Möglichkeiten und Entwicklungslinien einer Institutionalisierung 1850-1933, Berlin, Duncker und Humblot, 1994.

Seidlitz 1905 : Woldemar von Seidlitz, «Ein deutsches Museum für asiatische Kunst », Museumskunde, I, 1905, p. 181-198.

Weigelt 2006 : Ute Weigelt, Lucian Scherman (1864-1946) und das Münchner Museum für Völkerkunde, Munich, Staatliches Museum für Völkerkunde, 2006.

Westphal-Hellbusch 1973 : Sigrid Westphal-Hellbusch, « Zur Geschichte des Museums », BässlerArchiv, N. F., vol. 21, 1973, pp. 1-101

Zimmerman 2006 : Andrew Zimmerman, « From natural science to primitive art », dans Cordula Grewe, Die Schau des Fremden, Austellungskonzepte zwischen Kunst, Kommerz und Wissenschaft, Stuttgart, Franz Steiner, 2006, p. 279-300. 
Zimmerman 2001 : Andrew Zimmerman, Anthropology and Antihumanism in Imperial Germany,

Chicago, The University of Chicago Press, 2001.

\section{NOTES}

1. Cité dans Westphal-Hellbusch 1973, p. 4. (Toutes les citations sont traduites de l'allemand par l'auteur)

2. Schmalenbach 1961 et Goldwater 1967.

3. Scheffler 1921, p. 20.

4. Les premiers plans sont présentés à Hambourg en 1904, à Munich en 1912.

5. Penny 2002, p. 144 et suiv.

6. Richter 1906, p. 214.

7. Zimmerman 2006, p. 286

8. Cité dans Westphal-Hellbusch 1973, p. 18.

9. Dr. Hauptmann, adresse à la chambre des députés de Prusse, 26 avril 1904, cité dans WestphalHellbusch, 1973, p. 21.

10. Krause 1928, p. 67.

11. Zimmermann 2001 p. 287-289.

12. Grosse 1905, p. 133.

13. Sur les aménagements de Scherman à Munich, voir Gareis 1990 ; Smolka, 1994 ; Weigelt 2006.

14. Cité par Weigelt 2006, p. 70.

15. Voir Gareis et Migge 1967.

16. Siebold (von) (1796-1866), médecin, fondateur de la première collection ethnographique ouverte au public en Europe, à Leiden en 1837, qui posa les fondements du musée royal d'Ethnologie des Pays-Bas, aujourd'hui Rijksmuseum voor Volkenkunde.

17. Grosse 1905.

18. Seidlitz 1905, p. 186.

19. La notion de Kultur est fondamentale dans l'ethnologie allemande : voir Zimmerman 2001.

20. Krämer 1904.

21. Gräbner 1908.

22. Lettre de Scherman à Albert Le Coq, 12 mai 1922, citée par Weigelt, p. 165.

23. Voir Rolland 2008.

24. Discours d'inauguration de Lucian Scherman en 1926, rapporté par Krause 1920, p. 68.

25. Lettre de Scherman à Le Coq, $1^{\mathrm{er}}$ mai 1922, citée par Weigelt, p. 167.

26. Scherman 1922.

27. Les deux articles fondateurs de ce courant, connu sous le nom de Kulturkreislehre, sont ceux publiés par Fritz Gräbner et Bernhard Ankermann dans la revue Museumskunde en 1905 (Gräbner 1905 et Ankermann 1905).

28. Description reprise de Gareis 1990, p. 111.

29. Heydrich 1930, p. 128.

30. Krause 1920, p. 67.

31. Frese 1960, p. 33. 


\section{RÉSUMÉS}

$\mathrm{Au}$ tournant du siècle, pour toucher un public plus large, les ethnologues responsables des musées d'ethnologie allemands renouvellent la présentation des collections. Ils s'interrogent alors sur la valeur artistique des objets et sur la place de l'art dans les musées d'ethnologie. La complexité des débats et la diversité des solutions proposées sont illustrées par les évolutions très différentes des musées de Berlin et de Munich dans les premières décennies du $\mathrm{xx}^{\mathrm{e}}$ siècle. Les collections du musée d'ethnologie de Berlin sont scindées suivant des critères esthétiques, tandis qu'une partie des objets asiatiques rejoignent un musée d'art asiatique. Le musée de Munich, rénové par Lucian Scherman à partir de 1907, oppose à cette double séparation entre art et ethnologie une vision globale des cultures du monde, tentant de concilier exigences scientifiques et mise en valeur artistique. Ces conceptions divergentes, qui émergent à la même époque partout en Europe, ouvrent les débats incessants du $\mathrm{xx}^{\mathrm{e}}$ siècle sur le statut des collections extraeuropéennes.

\section{INDEX}

Mots-clés : Adolf Bastian, Allemagne, anthropologie, art, arts décoratifs, arts d'Asie, arts primitifs, Berlin, esthétique, Lucian Scherman, Munich, muséographie, Schausammlung, Studiensammlung, Völkerkunde

\section{AUTEUR}

\section{ANNE-SOLÈNE ROLLAND}

Ancienne élève de l'École nationale supérieure, Anne-Solène Rolland est conservatrice du patrimoine, responsable de l'unité patrimoniale des collections textiles au musée du quai Branly. Elle prépare également un doctorat en études germaniques à l'université Paris-III intitulé Naissance et développement des musées d'ethnologie en Allemagne (1860-1933). Elle a coordonné récemment deux ouvrages sur l'histoire des musées : Les Musées de la Nation et De nouveaux modèles de musées? (L'Harmattan, Paris, 2008). 